\title{
Socle pairings on tautological rings
}

\author{
Felix Janda and Aaron Pixton
}

\begin{abstract}
We study some aspects of the $\lambda_{g}$-pairing on the tautological ring of $M_{g}^{c}$, the moduli space of genus $g$ stable curves of compact type. We consider pairing $\mathcal{K}$-classes with pure boundary strata, all tautological classes supported on the boundary, or the full tautological ring. We prove that the rank of this restricted pairing is equal in the first two cases and has an explicit formula in terms of partitions, while in the last case the rank increases by precisely the rank of the $\lambda_{g} \lambda_{g-1^{-}}$ pairing on the tautological ring of $M_{g}$.
\end{abstract}

Keywords. Tautological ring, kappa ring, Gorenstein conjecture, moduli of curves

2010 Mathematics Subject Classification. 14H10

\section{[Français]}

Titre. Accouplements-socles sur les anneaux tautologiques

Résumé. Nous étudions certains aspects de l'accouplement $\lambda_{g}$ sur l'anneau tautologique de $M_{g}^{c}$, l'espace de modules des courbes stables de type compact de genre $g$. Nous considérons l'accouplement de classes $\kappa$ avec des strates pures du bord, avec toutes les classes tautologiques supportées sur le bord ou bien avec l'anneau tautologique dans sa totalité. Nous montrons que le rang de cet accouplement restreint est le même dans les deux premiers cas et a une expression explicite en termes de partitions, tandis que dans le dernier cas, le rang augmente précisément du rang de l'accouplement $\lambda_{g} \lambda_{g-1}$ sur l'anneau tautologique de $M_{g}$.

Received by the Editors on July 11, 2017, and in final form on February 15, 2018. Accepted on February 9, 2019.

Felix Janda

Department of Mathematics, University of Michigan, 2074 East Hall, 530 Church Street, Ann Arbor, MI 48109, USA e-mail: janda@umich.edu

Aaron Pixton

Department of Mathematics, Massachusetts Institute of Technology, Cambridge, MA 02139, USA

e-mail: apixton@mit.edu 


\section{Contents}

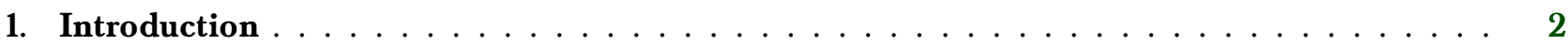

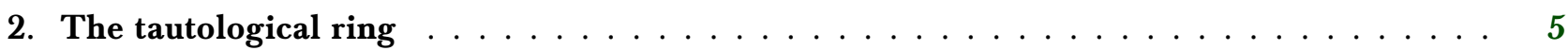

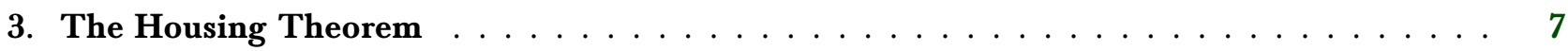

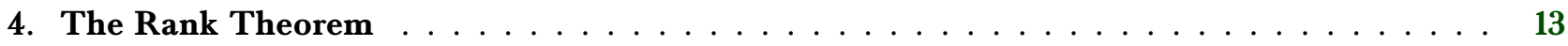

\section{Introduction}

Let $M_{g, n}$ be the moduli space of smooth curves of genus $g$ with $n$ marked points and let $\bar{M}_{g, n}$ be the Deligne-Mumford compactification, the moduli space of stable $n$-pointed nodal curves of arithmetic genus $g$. Inside this, let $M_{g, n}^{c}$ be the subspace of stable pointed curves of compact type, i.e. curves whose dual graph is a tree.

The intersection theory of these moduli spaces of curves is a subject of fundamental importance in algebraic geometry. When studying the Chow ring $A^{*}\left(\bar{M}_{g, n}\right)$, one is naturally led to consider a subring consisting of the classes such as the Arbarello-Cornalba $\mathcal{K}$-classes that are defined via certain tautological maps between the $\bar{M}_{g, n}$. This subring is the tautological ring $R^{*}\left(\bar{M}_{g . n}\right)$. Tautological rings $R^{*}\left(M_{g, n}\right)$ and $R^{*}\left(M_{g, n}^{c}\right)$ for $M_{g, n}$ and $M_{g, n}^{c}$ can be defined by restriction. We will primarily be interested in $R^{*}\left(M_{g}^{c}\right)$, the case of compact type with no marked points.

Inside $R^{*}\left(M_{g, n}^{c}\right)$ there is the subring $\kappa^{*}\left(M_{g, n}^{c}\right)$ generated by the $\kappa$-classes $\kappa_{1}, \kappa_{2}, \ldots$. The kappa ring $\mathcal{K}^{*}\left(M_{g, n}^{c}\right)$ has been studied in detail by Pandharipande [6]. In particular, for $n>0$ a complete description of the kappa ring is given. For this reason we concentrate on the case $n=0$ in this paper.

When restricted to the moduli space of smooth curves $M_{g}$, the tautological ring $R^{*}\left(M_{g}\right)$ is actually equal to the kappa ring $\kappa^{*}\left(M_{g}\right)$. This means that on $M_{g}^{c}$, any tautological class can be written as the sum of a polynomial in the $\mathcal{K}$-classes and a class supported on the boundary. We denote by $B R^{*}\left(M_{g}^{c}\right)$ the ideal of tautological classes supported on the boundary, so the tautological ring $R^{*}\left(M_{g}^{c}\right)$ is linearly spanned by $\kappa^{*}\left(M_{g}^{c}\right)$ and $B R^{*}\left(M_{g}^{c}\right)$.

A general element of $B R^{*}\left(M_{g}^{c}\right)$ is a linear combination of classes obtained by taking the pushforward of tautological classes via gluing maps

$$
M_{g_{1}, n_{1}}^{c} \times M_{g_{2}, n_{2}}^{c} \times \cdots \times M_{g_{k}, n_{k}}^{c} \rightarrow M_{g}^{c} .
$$

When the class 1 is pushed forward along such a map, this construction gives a pure boundary stratum. We let $P B R^{*}\left(M_{g}^{c}\right)$ denote the linear subspace of $B R^{*}\left(M_{g}^{c}\right)$ generated by the pure boundary strata.

There are natural bilinear pairings

$$
\begin{aligned}
R^{r}\left(M_{g}^{c}\right) \times R^{2 g-3-r}\left(M_{g}^{c}\right) \rightarrow R^{2 g-3}\left(M_{g}^{c}\right) \cong \mathbb{Q}, \\
R^{r}\left(M_{g}\right) \times R^{g-2-r}\left(M_{g}\right) \rightarrow R^{g-2}\left(M_{g}\right) \cong \mathbb{Q},
\end{aligned}
$$

given by the product in the Chow ring and the socle evaluations. These pairings are called the $\lambda_{g}$ - and $\lambda_{g} \lambda_{g-1}$-pairings respectively because they may be defined by integrating against these classes in $\bar{M}_{g}$.

In this paper we will study the restriction

$$
\kappa^{d}\left(M_{g}^{c}\right) \times R^{r}\left(M_{g}^{c}\right) \rightarrow \mathbb{Q}
$$

of the $\lambda_{g}$-pairing for $r+d=2 g-3$, for any $g \geq 2$. The following theorems, our main results, were previously conjectured by Pandharipande. 
Housing Theorem. The rank of the $\lambda_{g}$-pairing of $\kappa$-classes against boundary classes

$$
\kappa^{d}\left(M_{g}^{c}\right) \times B R^{r}\left(M_{g}^{c}\right) \rightarrow \mathbb{Q}
$$

equals the rank of the $\lambda_{g}$-pairing of $\kappa$-classes against pure boundary strata

$$
\kappa^{d}\left(M_{g}^{c}\right) \times P B R^{r}\left(M_{g}^{c}\right) \rightarrow \mathbb{Q} .
$$

Furthermore, these ranks are equal to the number of partitions of $d$ of length less than $r+1$ plus the number of partitions of $d$ of length $r+1$ which contain at least two even parts.

Rank Theorem. The rank of the $\lambda_{g}$-pairing of $\kappa$-classes against general tautological classes

$$
\kappa^{d}\left(M_{g}^{c}\right) \times R^{r}\left(M_{g}^{c}\right) \rightarrow \mathbb{Q}
$$

equals the sum of the rank of the $\lambda_{g}$-pairing of $\mathcal{\kappa}$-classes against boundary classes

$$
\kappa^{d}\left(M_{g}^{c}\right) \times B R^{r}\left(M_{g}^{c}\right) \rightarrow \mathbb{Q}
$$

and the rank of the $\lambda_{g} \lambda_{g-1}$-pairing

$$
\kappa^{r}\left(M_{g}\right) \times \kappa^{g-2-r}\left(M_{g}\right) \rightarrow \mathbb{Q} .
$$

These theorems will be proven by direct combinatorial analysis of the well known formulae for calculating the integrals arising in the pairings. In particular, we have no geometric explanation of the Rank Theorem, which connects the compact-type case and the smooth case.

\section{A. Consequences}

It has been conjectured by Faber [1] that $\mathcal{K}^{*}\left(M_{g}\right)=R^{*}\left(M_{g}\right)$ is a Gorenstein ring with socle in degree $g-2$. He verified this for $g \leq 23$ by computing many relations between the $\mathcal{K}$-classes and checking that they produced a Gorenstein ring. However, starting in genus 24, the known methods of producing relations have failed to give enough relations to yield a Gorenstein ring. In fact, the known relations have all been in the span of the Faber-Zagier (FZ) relations, and these relations produce a Gorenstein ring if and only if $g \leq 23$.

There are therefore mystery relations in $R^{*}\left(M_{g}\right)$ : formal polynomials in $\mathcal{K}$-classes which pair to zero with any $\mathcal{K}$ polynomial in $R^{*}\left(M_{g}\right)$ of complementary degree but are not a linear combination of FZ relations. If one assumes Faber's Gorenstein conjecture then these relations must hold in $R^{*}\left(M_{g}\right)$. Since FZ relations extend to tautological relations in $R^{*}\left(\bar{M}_{g}\right)$ (this is a consequence of the proof of the FZ relations in [7]), a possible reason for the existence of mystery relations might be if they do not extend tautologically to $R^{*}\left(M_{g}^{c}\right)$ or $R^{*}\left(\bar{M}_{g}\right)$. The Rank Theorem can be interpreted as saying that part of the obstruction to this extension is zero: the mystery relations at least extend to classes in the tautological ring of $M_{g}^{c}$ which pair to zero with the $\mathcal{K}$ subring. It is an interesting question whether the mystery relations extend to classes in the tautological ring of $M_{g}^{c}$ which are relations in the Gorenstein quotient (i.e. pair to zero with the entire tautological ring).

In [6] Pandharipande gives a minimal set of generators of $\mathcal{K}^{*}\left(M_{g, n}^{c}\right)$ for $n>0$ and relates higher genus relations to genus 0 relations. More precisely, he shows that there is a surjective (graded) ring homomorphism

$$
\kappa^{*}\left(M_{0,2 g+n}^{c}\right) \stackrel{l_{g, n}}{\rightarrow} \mathcal{K}^{*}\left(M_{g, n}^{c}\right),
$$

which is an isomorphism for $n \geq 1$, or in degrees up to $g-2$ when $n=0$. The Rank Theorem gives us information about the $n=0$ case in higher degrees. 
Theorem 1. Let $g \geq 2,0 \leq e \leq g-2$, and $d=g-1+e$. Let $\delta_{d}$ be the rank of the kernel of the map from $\kappa^{d}\left(M_{g}^{c}\right)$ to the Gorenstein quotient of $R^{*}\left(M_{g}^{c}\right)$. Let $\gamma_{e}$ be the rank of the space of $\kappa$-relations of degree $e$ in the Gorenstein quotient of $R^{*}\left(M_{g}\right)$. Let $N_{e}$ denote the number of partitions of e of length greater than $g-1-e$. Then the degree-d part of the kernel of $\iota_{g, 0}$ has rank $\gamma_{e}-\delta_{d}-N_{e}$.

Proof. We use the notation $|P(m)|$ for the number of partitions of $m$ and $|P(m, k)|$ for the number of partitions of $m$ of length at most $k$, so $N_{e}=|P(e)|-|P(e, g-1-e)|$. By [6], the rank of $\kappa^{d}\left(M_{0,2 g}^{c}\right)$ is equal to $|P(d, 2 g-2-d)|$. On the other side, the rank of $\kappa^{d}\left(M_{g}^{c}\right)$ is equal to $\delta_{d}$ plus the rank of the first pairing appearing in the Rank Theorem. The rank of the second pairing appearing in the Rank Theorem is given by the Housing Theorem and is equal to $|P(d, 2 g-2-d)|-X$, where $X$ is the number of partitions of $d$ of length $2 g-2-d$ with no even parts. By subtracting one from each part and dividing by two, we have that $X=|P(e, g-1-e)|$. The rank of the third pairing appearing in the Rank Theorem is equal to $|P(e)|-\gamma_{e}$. Putting all these pieces together gives

$$
\begin{aligned}
& \operatorname{dim}_{\mathbb{Q}} \kappa^{d}\left(M_{0,2 g}^{c}\right)-\operatorname{dim}_{\mathbb{Q}} \kappa^{d}\left(M_{g}^{c}\right) \\
& =|P(d, 2 g-2-d)|-\left(\delta_{d}+|P(d, 2 g-2-d)|-|P(e, g-1-e)|+|P(e)|-\gamma_{e}\right) \\
& =\gamma_{e}-\delta_{d}-N_{e},
\end{aligned}
$$

as desired.

Remark. The components $\gamma_{e}$ and $\delta_{d}$ appearing in the above theorem both have conjectural values. The FZ relations give a prediction for $\gamma_{e}$ (if they are the only relations in the first half of the Gorenstein quotient and are linearly independent):

$$
\gamma_{e}= \begin{cases}a(3 e-g-1) & \text { if } e \leq \frac{g-2}{2} \\ a(3(g-2-e)-g-1)+|P(e)|-|P(g-2-e)| & \text { else, }\end{cases}
$$

where $a(n)$ is the number of partitions of $n$ with no parts of sizes $5,8,11,14, \ldots$

Although the Gorenstein conjecture in compact type is false (see [8]), it is still reasonable to predict that the kernel of the map from $R^{d}\left(M_{g}^{c}\right)$ to its Gorenstein quotient will fail to intersect the relatively small subring $\kappa^{d}\left(M_{g}^{c}\right)$. This would imply that $\delta_{d}=0$. Combining this prediction with the FZ prediction for $\gamma_{e}$ gives a conjecture for all the Betti numbers of $\kappa^{*}\left(M_{g}^{c}\right)$ : we expect that

$$
\operatorname{dim}_{\mathbb{Q}} \kappa^{d}\left(M_{g}^{c}\right)=|P(d, 2 g-2-d)|-a(3 d-4 g+2) \quad \text { if } 0 \leq d \leq \frac{3 g-2}{2},
$$

along with a slightly more complicated formula in the case $\frac{3 g-2}{2}<d \leq 2 g-3$.

\section{B. Plan of the paper}

In Section 2, we review basic facts about the tautological ring. In Section 3, we prove the Housing Theorem. Finally, in Section 4, we state and prove a slightly more explicit version of the Rank Theorem (see Theorem 2).

\section{Acknowledgments}

The first named author wants to thank his advisor Rahul Pandharipande for the introduction to this topic and various discussions. The beginning of Section 1.A elaborates an email from him.

The first named author was supported by the Swiss National Science Foundation grant SNF 200021_143274. The second named author was supported by an NDSEG graduate fellowship. 


\section{The tautological ring}

\section{A. Tautological Classes}

The subrings $R^{*}\left(\bar{M}_{g . n}\right)$ of tautological classes in the Chow rings $A^{*}\left(\bar{M}_{g, n}\right)$ are collectively defined as the smallest subrings which are closed under pushforward via the maps forgetting markings $\bar{M}_{g, n} \rightarrow \bar{M}_{g, n-1}$ and the gluing maps

$$
\begin{gathered}
\bar{M}_{g_{1}, n_{1} \sqcup\{\star\}} \times \bar{M}_{g_{2}, n_{2} \sqcup\{\bullet\}} \rightarrow \bar{M}_{g_{1}+g_{2}, n_{1}+n_{2}} \\
\text { and } \\
\bar{M}_{g, n \sqcup\{\star, \bullet\}} \rightarrow \bar{M}_{g+1, n}
\end{gathered}
$$

defined by gluing together $\star$ and $\bullet$. It turns out that nearly all classes on the moduli space of curves that appear naturally in geometry lie in the tautological ring.

For each $i=1,2, \ldots, n$, there is a line bundle $\mathbb{L}_{i}$ on $\bar{M}_{g, n}$ given by the cotangent space at the $i$ th marked point. The first Chern classes of these line bundles are denoted by $\psi_{i}=c_{1}\left(\mathbb{L}_{i}\right) \in A^{1}\left(\bar{M}_{g, n}\right)$. The $\mathcal{\kappa}$-classes are then pushforwards of powers of the $\psi$ classes:

$$
\kappa_{m}=\pi_{*}\left(\psi_{n+1}^{m+1}\right) \in A^{m}\left(\bar{M}_{g, n}\right),
$$

where $\pi$ is the forgetful map $\bar{M}_{g, n+1} \rightarrow \bar{M}_{g, n}$.

It is well known (see e.g. [5]) that the $\kappa$ - and $\psi$-classes combined with pushforward by the gluing morphisms alone are sufficient to generate the tautological rings. In other words, $R^{*}\left(\bar{M}_{g, n}\right)$ is additively generated by classes of the form

$$
\xi_{\Gamma *}\left(\prod_{v \text { vertex of } \Gamma} \theta_{v}\right),
$$

where $\Gamma$ is a stable graph expressing the data of the gluing map

$$
\xi_{\Gamma}: \prod_{v \text { vertex of } \Gamma} \bar{M}_{g(v), n(v)} \rightarrow \bar{M}_{g, n}
$$

and the $\theta_{v} \in R^{*}\left(\bar{M}_{g(v), n(v)}\right)$ are arbitrary monomials in the $\psi$ - and $\mathcal{K}$-classes.

The tautological rings $R^{*}\left(M_{g, n}^{c}\right)$ and $R^{*}\left(M_{g, n}\right)$ are defined as the image of $R^{*}\left(\bar{M}_{g, n}\right)$ under restriction. In the case of $R^{*}\left(M_{g, n}^{c}\right)$, this means that the stable graph $\Gamma$ must be a tree, while $R^{*}\left(M_{g, n}\right)$ is simply the subring of polynomials in the $\mathcal{K}$ - and $\psi$-classes.

The ring $R^{*}\left(M_{g, n}^{c}\right)$ has one-dimensional socle, in degree $2 g-3+n$ :

$$
R^{2 g-3+n}\left(M_{g, n}^{c}\right) \cong \mathbb{Q}
$$

This gives a canonical (up to scaling) bilinear pairing on $R^{*}\left(M_{g, n}^{c}\right)$, which can be realized explicitly by integrating against the Hodge class $\lambda_{g}$ :

$$
R^{*}\left(M_{g, n}^{c}\right) \times R^{*}\left(M_{g, n}^{c}\right) \rightarrow \mathbb{Q}, \quad(\alpha, \beta) \mapsto \int_{\bar{M}_{g, n}} \alpha \beta \lambda_{g} .
$$

Here, the integral is defined by taking any extensions of $\alpha$ and $\beta$ to $R^{*}\left(\bar{M}_{g, n}\right)$. It is independent of which particular extension one has chosen because $\lambda_{g}$ vanishes on the complement of $M_{g, n}^{c}$.

The $\lambda_{g} \lambda_{g-1}$-pairing is a similar pairing for the moduli space of smooth curves, given by

$$
R^{*}\left(M_{g}\right) \times R^{*}\left(M_{g}\right) \rightarrow \mathbb{Q}, \quad(\alpha, \beta) \mapsto \int_{\bar{M}_{g}} \alpha \beta \lambda_{g} \lambda_{g-1}
$$

Notice that the $\lambda_{g}$-pairing on $R^{*}\left(M_{g}^{c}\right)$ vanishes above degree $2 g-3$ whereas the $\lambda_{g} \lambda_{g-1}$-pairing on $R^{*}\left(M_{g}\right)$ already vanishes above degree $g-2$. 


\section{B. Notation concerning partitions}

In the following sections we will use the following notation heavily. A partition $\sigma$ is an unordered collection of natural numbers (a multiset). We call its elements parts. Its size is the sum of all its parts. The length $\ell(\sigma)$ of a partition $\sigma$ is the number of parts in it. For natural numbers $n$ and $r$, we denote by $P(n)$ the set of partitions of size $n$ and by $P(n, r)$ the set of partitions of size $n$ and length at most $r$. Furthermore, let $I(\sigma)$ be a set of $\ell(\sigma)$ elements which we will use to index the parts of $\sigma$. For example we could take

$$
I(\sigma)=[\ell(\sigma)]:=\{1, \ldots, \ell(\sigma)\} .
$$

For two partitions $\sigma, \tau \in P(n)$ and a map $\varphi: I(\sigma) \rightarrow I(\tau)$ we say that $\varphi$ is a refining function of $\tau$ into $\sigma$ if for any $i \in I(\tau)$ we have

$$
\tau_{i}=\sum_{j \in \varphi^{-1}(i)} \sigma_{j}
$$

If for given $\sigma$ and $\tau$ there exists a refining function $\varphi$ of $\tau$ into $\sigma$, we say that $\sigma$ is a refinement of $\tau$.

For a finite set $S$, a set partition $P$ of $S$ (written $P \vdash S$ ) is a set $P=\left\{S_{1}, \ldots, S_{m}\right\}$ of nonempty subsets of $S$ such that $S$ is the disjoint union of the $S_{i}$.

For a partition $\sigma$ and a set $S$ of subsets of $I(\sigma)$ we define a new partition $\sigma^{S}$ indexed by the elements of $S$ by setting $\left(\sigma^{S}\right)_{s}=\sum_{i \in S} \sigma_{i}$ for each $s \in S$. Usually we will take a set partition $P$ of $I(\sigma)$ for $S$. For a subset $T \subseteq I(\sigma)$, we define the restriction $\left.\sigma\right|_{T}$ of $\sigma$ to $T$ by $\sigma^{S}$, where $S$ is the set of all 1-element subsets of $T$; in other words, $\left.\sigma\right|_{T}=\left(\sigma_{t}\right)_{t \in T}$.

\section{C. Integral calculations}

The basic formula for the evaluation of the integrals arising in the $\lambda_{g}$-pairing is (see [3])

$$
\int_{\bar{M}_{g, n}} \prod_{i=1}^{n} \psi_{i}^{\tau_{i}} \lambda_{g}=\left(\begin{array}{c}
2 g-3+n \\
\tau
\end{array}\right) \int_{\bar{M}_{g, 1}} \psi_{1}^{2 g-2} \lambda_{g},
$$

where $\tau_{1}, \ldots, \tau_{n}$ are nonnegative integer numbers with sum $2 g-3+n$. The formula is symmetric with respect to the sorting of the markings and hence we only need to know the partition corresponding to $\tau$ in order to calculate these integrals. Since we are only interested in the ranks of the pairing, the only thing we will need to know about the integral on the right hand side is that it is nonzero (see [2]).

We will need to evaluate integrals involving $\psi$-classes as part of the proof of the housing theorem. However our main interest lies in the calculation of integrals involving $\mathcal{K}$-classes. Using the definition of the $\mathcal{K}$-classes as push-forwards of powers of $\psi$-classes, we can find a nice expression for the quotients

$$
\vartheta(\sigma ; \tau):=\left(\int_{\bar{M}_{g, \ell(\tau)}} \kappa_{\sigma} \psi^{\tau} \lambda_{g}\right)\left(\int_{\bar{M}_{g, 1}} \psi_{1}^{2 g-2} \lambda_{g}\right)^{-1} .
$$

In this equation we have used $\kappa_{\sigma}$ as an abbreviation for $\prod_{i \in I(\sigma)} \kappa_{\sigma_{i}}$ and $\psi^{\tau}$ for $\prod_{i \in I(\tau)} \psi_{i}^{\tau_{i}}$ indexing the $|\tau|$ marked points by the parts of $\tau$. We will write

$$
\vartheta(\sigma):=\vartheta(\sigma ; \emptyset)
$$

when we just have $\mathcal{K}$-classes and no $\psi$-classes.

Lemma 1. For partitions $\sigma$ and $\tau$ such that $2 g-3+\ell(\tau)=|\sigma|+|\tau|$, we have

$$
\vartheta(\sigma ; \tau)=\sum_{P \vdash I(\sigma)}(-1)^{|P|+\ell(\sigma)}\left(\begin{array}{c}
2 g-3+|P|+\ell(\tau) \\
\left(\left(\sigma^{P}\right)_{i}+1\right)_{i \in P}, \tau
\end{array}\right) .
$$


Proof. From the basic socle evaluation formula we see that it suffices to prove the identity

$$
\kappa_{\sigma} \psi^{\tau} \lambda_{g}=\sum_{P \vdash I(\sigma)}(-1)^{|P|+\ell(\sigma)} \pi_{*}\left(\psi^{\left(\left(\sigma^{P}\right)_{i}+1\right)_{i \in P}} \psi^{\tau} \lambda_{g}\right) .
$$

in the Chow ring $R\left(\bar{M}_{g, \ell(\tau)}\right)$, where by abuse of notation $\pi$ is the forgetful map $\bar{M}_{g, \ell(\tau)+n} \rightarrow \bar{M}_{g, \ell(\tau)}$ for the appropriate $n$. Since $\pi^{*}\left(\lambda_{g}\right)=\lambda_{g}$, we can further reduce to

$$
\kappa_{\sigma} \psi^{\tau}=\sum_{P \vdash I(\sigma)}(-1)^{|P|+\ell(\sigma)} \pi_{*}\left(\psi^{\left(\left(\sigma^{P}\right)_{i}+1\right)_{i \in P}} \psi^{\tau}\right) .
$$

This follows from the pushforward formula

$$
\pi_{*}\left(\psi^{\left(\sigma_{i}+1\right)_{i \in P}} \psi^{\tau}\right)=\sum_{P \vdash I(\sigma)}\left(\prod_{S \in P}(|S|-1) !\right) \kappa_{\sigma^{P}} \psi^{\tau} .
$$

and partition refinement inversion.

To evaluate the more general integrals which arise when we pair $\mathcal{\kappa}$-classes with arbitrary tautological classes, we can restrict ourselves to pairing a $\mathcal{K}$-monomial with the additive set of generators described in Section 2.A. In this case, we have to sum over the set of possible distributions of the $\mathcal{K}$-classes to the vertices of $\Gamma$ and then multiply the $\lambda_{g}$-integrals at each vertex.

The $\lambda_{g} \lambda_{g-1}$-pairing formula is similar:

$$
\int_{\bar{M}_{g, n}} \psi^{\sigma} \lambda_{g} \lambda_{g-1}=\frac{(2 g-3+\ell(\sigma)) !(2 g-1) ! !}{(2 g-1) ! \prod_{i \in I(p)}\left(2 \sigma_{i}+1\right) ! !} \int_{\bar{M}_{g}} \psi^{g-2} \lambda_{g} \lambda_{g-1} .
$$

The integral on the right hand side is known to be nonzero (see [4]). We can calculate the $\kappa$-integrals analogously to Lemma 1.

\section{The Housing Theorem}

\section{A. Housing Partitions}

Let us now study pairing $\kappa$-monomials of degree $d$ with pure boundary classes via the $\lambda_{g}$-pairing. Each pure boundary stratum in codimension $2 g-3-d$ is determined by a tree $\Gamma=(V, E)$ with $|V|=2 g-2-d$ vertices and $|E|=2 g-3-d$ edges, and a genus function $g: V \rightarrow \mathbb{Z}_{\geq 0}$ with $\sum_{v \in V} g(v)=g$. Then, the class is the push-forward of 1 along the gluing map $\xi_{\Gamma}: \prod_{v \in V} M_{g(v), n(v)}^{c} \rightarrow M_{g}^{c}$ corresponding to the tree $\Gamma$, where $n(v)$ is the degree of the vertex $v$. From this data, we obtain a partition of

$$
\begin{aligned}
\sum_{v \in V}(2 g(v)-3+n(v)) & =2 g-3(2 g-2-d)+2(2 g-3-d) \\
& =d
\end{aligned}
$$

by collecting the socle dimensions $2 g(v)-3+n(v)$ for each vertex $v \in V$ and throwing away the zeroes. We will call this partition the housing data of the pure boundary stratum. From the $\lambda_{g}$ formula, it is easy to see that the pairing of the $\mathcal{K}$-ring with a pure boundary stratum is determined by its housing data.

On the other hand, it is interesting to consider which partitions of $d$ can arise as housing data corresponding to a pure boundary stratum. We will call these partitions housing partitions.

Lemma 2. A partition $\sigma$ of $d$ is a housing partition if and only if it either has fewer than $2 g-2-d$ parts or exactly $2 g-2-d$ parts, at least two of which are even. 
Proof. Only partitions of length at most $2 g-2-d$ can be housing partitions because there are only that many vertices. Furthermore, it is easy to see that no partition of $2 g-2-d$ parts with fewer than two even parts can arise since every vertex with only one edge gives an even part (or no part if $g(v)=1$ ).

Now suppose $\sigma$ is a partition of $d$ with either fewer than $2 g-2-d$ parts or exactly $2 g-2-d$ parts with at least two even. Let $\left(\tau_{i}\right)_{1 \leq i \leq 2 g-2-d}$ be the tuple of nonnegative integers given by appending $2 g-2-d-\ell(\sigma)$ zeroes to $\sigma$, so that the sum of the $\tau_{i}$ is $d$, and exactly $2 k+2$ of the $\tau_{i}$ are even for some nonnegative integer $k$.

Construct a tree $\Gamma$ by taking a path of $2 g-2-d-k$ vertices and adding $k$ additional leaves connected to vertices $2,3, \ldots, k+1$ along the path respectively. Thus, $\Gamma$ has $2 g-2-d$ vertices, each of degree at most three, and exactly $2 k+2$ of the vertices of $\Gamma$ have odd degree. We now choose a bijection between the $\tau_{i}$ and the vertices of $\Gamma$ such that even $\tau_{i}$ are assigned to vertices of odd degree. We can then assign a genus $g_{i}=\left(\tau_{i}+3-n_{i}\right) / 2$ to each vertex, where $n_{i}$ is the degree of the vertex to which $\tau_{i}$ was assigned. The resulting stable tree has housing data $\sigma$, as desired.

\section{B. Reduction to a combinatorial problem}

We have already described the housing data of a pure boundary stratum. Let us now describe a similar notion for any class in the generating set described in Section 2.A. Such a class is given by a boundary stratum corresponding to a tree $\Gamma=(V, E)$ and a genus assignment $g: V \rightarrow \mathbb{Z}_{\geq 0}$, along with assignments of monomials in $\kappa$ - and $\psi$-classes (of degrees $r(v)$ and $s(v)$ respectively) to each component of the stratum. Let $k=\sum_{v \in V}(r(v)+s(v))$; then we must have $|E|=2 g-3-d-k$ edges in the tree in order to obtain a class of degree $2 g-3-d$. If this class does not vanish by dimension reasons, then we can obtain a partition $\gamma$ of

$$
\sum_{v \in V}(2 g(v)-3+n(v)-r(v)-s(v))=2 g-3(2 g-2-d-k)+2(2 g-3-d-k)-k=d
$$

by assigning to each vertex of $V$ the number $2 g(v)-3+n(v)-r(v)-s(v)$. This is exactly the degree $d^{\prime}(v)$ such that the $\lambda_{g(v)}$-pairing of $R^{d^{\prime}(v)}\left(M_{g(v), n(v)}^{c}\right)$ with the monomial of $\psi$ - and $\kappa$-classes at $v$ is not zero for dimension reasons. Then, the pairing with the boundary class is determined by the partition $\gamma$, an assignment of degrees $r(i)$ and $s(i)$ to the parts $i \in I(\gamma)$ and partitions $\tau_{i} \in P(r(i))$ and $\rho_{i} \in P(s(i))$ corresponding to the $\mathcal{K}$ - and $\psi$-monomials. In particular we can leave out classes which were assigned to vertices with $2 g(v)-3+n(v)-r(v)-s(v)=0$ and we do not need to remember which node corresponds to each $\psi$. The result of the $\lambda_{g}$-pairing of this class together with a $\kappa$-monomial corresponding to a partition $\pi$ of $d$ is (up to scaling) given by

$$
\sum_{\varphi} \prod_{j \in I(\gamma)} \vartheta\left(\pi_{\varphi^{-1}(j)}, \tau_{j} ; \rho_{j}\right)
$$

where the sum runs over all refining functions $\varphi$ of $\gamma$ into $\pi$.

When we view $\mathbb{Q}^{P(d)}$ as a ring of formal $\kappa$-polynomials, this pairing gives linear forms

$$
v_{\gamma,\left\{\tau_{i}\right\},\left\{\rho_{i}\right\}} \in\left(\mathbb{Q}^{P(d)}\right)^{*} .
$$

We notice that the formulas still make combinatorial sense even if the triple $\left(\gamma,\left\{\tau_{i}\right\},\left\{\rho_{i}\right\}\right)$ does not come from pairing with an actual tautological class.

The special case where all the $r(i)$ and $s(i)$ are zero gives the pairing of $\kappa$ classes with pure boundary classes. We get $|P(d)|$ linear forms $M_{\lambda}$, which we normalize such that $M_{\lambda}(\lambda)=1$ :

$$
M_{\lambda}(\pi)=\frac{1}{\operatorname{Aut}(\lambda)} \sum_{\varphi} \prod_{j \in I(\lambda)} \vartheta\left(\pi_{\varphi^{-1}(j)}\right) .
$$

In this way we obtain a basis of $\left(\mathbb{Q}^{P(d)}\right)^{*}$. If we sort partitions in any way such that shorter partitions come before longer partitions, then the basis change matrix from this basis to the standard basis is triangular with ones on the diagonal. Note that this basis uses some partitions which are not housing partitions. 
The housing theorem can now be reformulated as follows:

Claim. The span of $\left\{M_{\lambda}: \lambda\right.$ is a housing partition $\}$ in $\left(\mathbb{Q}^{P(d)}\right)^{*}$ equals the span of the $v_{\gamma,\left\{\tau_{i}\right\},\left\{\rho_{i}\right\}}$ for all choices of housing data.

To prove this claim, we will first in Section 3.C express the vectors $v_{\gamma,\left\{\tau_{i}\right\},\left\{\rho_{i}\right\}}$ for any choice of housing data in terms of the basis of $\left(\mathbb{Q}^{P(d)}\right)^{*}$ we have described above. We will then in Section 3.D rewrite the coefficients as counts of certain combinatorial objects. This combinatorial interpretation is proved in Section 3.E. We conclude in Section 3.F by showing that when expressing vectors $v$ corresponding to actual housing data in terms of the $M_{\lambda}$, the coefficient is zero whenever $\lambda$ is not a housing partition.

\section{C. A Matrix Inversion}

In Section 3.B we have seen that there are formal expansions

$$
v_{\gamma,\left\{\tau_{i}\right\},\left\{\rho_{i}\right\}}=\sum_{\lambda \in P(d)} c_{\lambda, \gamma,\left\{\tau_{i}\right\},\left\{\rho_{i}\right\}} M_{\lambda}
$$

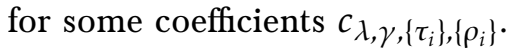

We can calculate $c_{\lambda, \gamma,\left\{\tau_{i}\right\},\left\{\rho_{i}\right\}}$ explicitly by inverting the triangular matrix given by equation (1). We obtain

$$
c_{\lambda, \gamma,\left\{\tau_{i}\right\},\left\{\rho_{i}\right\}}=\sum_{l=0}^{\infty}(-1)^{l} \sum_{\lambda_{0} \stackrel{\varphi_{1}}{\rightarrow} \stackrel{\varphi_{l}}{\rightarrow} \lambda_{l} \stackrel{\varphi_{l+1}}{\rightarrow} \gamma} \frac{v_{\gamma,\left\{\tau_{i}\right\},\left\{\rho_{i}\right\}}\left(\lambda_{l}\right)}{\prod_{i=1}^{l}\left|\operatorname{Aut}\left(\lambda_{i}\right)\right|} \prod_{i=1}^{l} \prod_{j \in I\left(\lambda_{i}\right)} \vartheta\left(\left(\lambda_{i-1}\right)_{\varphi_{i}^{-1}(j)}\right),
$$

where we sum over chains $\lambda=\lambda_{0}, \ldots \lambda_{l}$ of refinements of $\gamma$ with corresponding refining functions $\varphi_{i}$. In particular, $c_{\lambda, \gamma,\left\{\tau_{i}\right\},\left\{\rho_{i}\right\}}=0$ if $\lambda$ is not a refinement of $\gamma$.

We can reduce to the special case in which $\gamma=(d)$ is of length one by splitting this sum based on the composition $\varphi:=\varphi_{l+1} \circ \varphi_{l} \circ \cdots \circ \varphi_{1}$ and examining the contribution of the preimages of the $j \in I(\gamma)$. The result is

$$
c_{\lambda, \gamma,\left\{\tau_{i}\right\},\left\{\rho_{i}\right\}}=\sum_{\varphi} \prod_{j \in I(\gamma)} c_{\lambda_{\varphi^{-1}(j)},}\left(\gamma_{j}\right),\left\{\tau_{j}\right\},\left\{\rho_{j}\right\},
$$

summed over refining functions $\varphi$ of $\gamma$ into $\lambda$.

When $\gamma=(d)$, we set $\tau_{1}=: \tau$ and $\rho_{1}=: \rho$, and we can write more compactly:

$$
c_{\lambda,(d),\{\tau\},\{\rho\}}=\sum_{l=0}^{\infty}(-1)^{l} \sum_{\substack{\lambda_{0} \rightarrow \ldots \varphi_{l} \\ \rightarrow}} \frac{\vartheta\left(\lambda_{l}, \tau ; \rho\right)}{\prod_{i=1}^{l}\left|\operatorname{Aut}\left(\lambda_{i}\right)\right|} \prod_{i=1}^{l} \prod_{j \in I\left(\lambda_{i}\right)} \vartheta\left(\left(\lambda_{i-1}\right)_{\varphi_{i}^{-1}(j)}\right)
$$

\section{D. Interpreting the coefficients combinatorially}

We will interpret the coefficients $c_{\lambda,(d),\{\tau\},\{\rho\}}$ as counting certain permutations of symbols labeled by the parts of the partitions $\lambda$, $\tau$, and $\rho$. We say that a symbol is of kind $i$ if it is labelled by some $i$ belonging to the disjoint union of the indexing sets of the partitions, $I(\lambda) \sqcup I(\tau) \sqcup I(\rho)$. There will in general be multiple symbols of a given kind.

Main Claim. The coefficient $c_{\lambda,(d),\{\tau\},\{\rho\}}$ counts the number of permutations of

- $\lambda_{i}+1$ symbols of kind $i$ for each $i \in I(\lambda)$,

- $\tau_{i}+1$ symbols of kind $i$ for each $i \in I(\tau)$, and 
- $\rho_{i}$ symbols of kind $i$ for each $i \in I(\rho)$

such that:

(1) If the last symbol of some kind $i$ is immediately followed by the first symbol of kind $j$ with $i, j \in I(\lambda) \sqcup I(\tau)$, then we have $i<j$.

(2) For $i \in I(\lambda)$, the last symbol of kind $i$ is not immediately followed by a symbol of kind $j$ for any $j \in I(\lambda)$, averaged over all total orders $<$ of $I(\lambda) \sqcup I(\tau)$ such that elements of $I(\tau)$ are smaller than elements of $I(\lambda)$.

It follows in particular that the coefficient $c_{\lambda, \gamma,\left\{\tau_{i}\right\},\left\{\rho_{i}\right\}}$ is non-negative.

\section{E. Proof of the Main Claim}

\section{E.a. Refinements of permutations of symbols}

For given natural numbers $d, n$ and a partition $\tau \in P(d)$, we will study permutations $S$ of $\tau_{i}+1$ symbols of kind $i$ for $i \in I(\tau)$ and $n$ symbols of kind $c$. (The permutations of symbols appearing in the previous section are an instance of this.) We will need to construct refined permutations of this type for partition refinements $\varphi: I(\sigma) \rightarrow I(\tau)$. For this, we need additional refinement data: for each $i \in I(\tau)$, let $T_{i}$ be a permutation of $\sigma_{j}+1$ symbols of kind $j$ for $j \in \varphi^{-1}(i)$.

Given $S$ and the refinement data, we can obtain a permutation $S^{\prime}$ of $\sigma_{i}+1$ symbols of kind $i$ and $n$ symbols of kind $c$ in the following way: For each $i \in I(\tau)$ and each $j \in \varphi^{-1}(i)$, modify $T_{i}$ by gluing the last symbol of kind $j$ with the immediately following symbol; the result is a permutation $T_{i}^{\prime}$ of $\tau_{i}+1$ symbols. To construct $S^{\prime}$ from $S$, for each $i$ we replace the symbols of kind $i$ by $T_{i}^{\prime}$ and then remove the glue.

\section{E.b. Reinterpretation}

We start with a combinatorial interpretation of the number $\vartheta(\sigma ; \tau)$ for partitions $\sigma$ and $\tau$.

Lemma 3. Given an arbitrary total order $<$ on $I(\sigma)$, the number $\vartheta(\sigma ; \tau)$ is equal to the number of permutations of

- $\sigma_{i}+1$ symbols of kind $i$ for each $i \in I(\sigma)$ and

- $\tau_{i}$ symbols of kind $i$ for each $i \in I(\tau)$

such that the following property holds: $i<j$.

If the last symbol of kind $i$ is immediately followed by the first symbol of kind $j$ for $i, j \in I(\sigma)$, then we have

Proof. For each permutation $S$ of symbols as above, but not necessarily satifying the property, we can assign a set partition $Q_{S} \vdash I(\sigma)$ which measures in what ways it fails to satisfy the property: $Q_{S}$ is the finest set partition such that if $i>j$ and the last symbol of kind $i$ is immediately followed by the first symbol of kind $j$ in $S$, then $i$ and $j$ are in the same part of $Q_{S}$. Thus $S$ satisfies the given property if and only if $Q_{S}$ is the set partition with all parts of size 1 .

The multinomial coefficient in the summand in the formula for $\vartheta(\sigma ; \tau)$ given by Lemma 1 corresponding to a set partition $P \vdash I(\sigma)$ counts the number of permutations $S$ such that for $p=\left\{p_{1}, \ldots, p_{k}\right\} \in P$ with $p_{1}>\cdots>p_{k}$, the last symbol of kind $p_{i}$ is immediately followed by the first symbol of kind $p_{i+1}$ in $S$ for $i=1, \cdots, k-1$. These are precisely the $S$ such that $Q_{S}$ can be obtained by combining parts of $P$ such that the largest element in one part is smaller than the smallest element of the other part.

This means that if we split the formula for $\vartheta(\sigma ; \tau)$ given by Lemma 1 into a sum over permutations $S$, the contribution of a permutation with failure set partition $Q=\left\{Q_{1}, \ldots, Q_{k}\right\}$ is precisely

$$
\prod_{i=1}^{k} \sum_{j=0}^{\left|Q_{k}\right|-1}(-1)^{j}\left(\begin{array}{c}
\left|Q_{k}\right|-1 \\
j
\end{array}\right),
$$

which is 1 for $Q$ the set partition with all parts of size 1 , and 0 otherwise. 
Equipped with Lemma 3, the next step in the proof of the Main Claim is to split the coefficient $c_{\lambda,(d),\{\tau\},\{\rho\}}$ into a sum over the set $S_{\lambda, \tau, \rho}$ of permutations of $\lambda_{i}+1$ (respectively, $\tau_{i}+1, \rho_{i}$ ) symbols of kind $i$ for $i \in I(\lambda)$ (respectively, $i \in I(\tau), i \in I(\rho)$ ). For this, we introduce the notion of the composite permutation.

Consider the following data:

- a chain of partitions $\lambda_{0}=\lambda, \lambda_{1}, \ldots, \lambda_{l}$ with refining maps $\varphi_{i}$ as in (3),

- the additional data of an order $<$ on $I\left(\lambda_{l}\right) \sqcup I(\tau)$ such that elements of $I(\tau)$ appear before elements of $I\left(\lambda_{l}\right)$,

- the additional data of orders on $\varphi_{i}^{-1}(j)$ for $1 \leq i \leq l$ and $j \in I\left(\lambda_{i}\right)$.

With this data, we identify each $\kappa$ socle evaluation factor

$$
\vartheta\left(\left(\lambda_{i-1}\right)_{\varphi_{i}^{-1}(j)}\right)
$$

with the number of permutations of $\left(\lambda_{i-1}\right)_{k}+1$ symbols of kind $k \in \varphi_{i}^{-1}(j)$ such that if the last symbol of kind $k$ is immediately followed by the first symbol of kind $k^{\prime}$, then $k<k^{\prime}$. We can interpret each such permutation as refinement data corresponding to the refining function $\varphi_{i}$ of $\lambda_{i+1}$ into $\lambda_{i}$.

Furthermore, we interpret the factor

$$
\vartheta\left(\lambda_{l}, \tau ; \rho\right)
$$

as the number of permutations $S_{l}$ of $\left(\lambda_{l}\right)_{k}+1, \tau_{k}+1$ and $\rho_{k}$ symbols of kind $k$ with $k \in I\left(\lambda_{l}\right), k \in I(\tau)$ and $k \in I(\rho)$, respectively, such that if the last symbol of kind $k$ is immediately followed by the first symbol of kind $k^{\prime}$ for $k, k^{\prime} \in I\left(\lambda_{l}\right) \sqcup I(\tau)$, then $k<k^{\prime}$.

Given all this data, we can build the composite permutation by repeatedly refining the collection of symbols of kind $k$ with $k \in I\left(\lambda_{l}\right)$ using the construction from Section 3.E.e and keeping the order of the other symbols intact. The result is a permutation of $\lambda_{k}+1, \tau_{k}+1$ and $\rho_{k}$ symbols of kind $k$ for $k \in I(\lambda)$, $k \in I(\tau)$ and $k \in I(\rho)$ respectively.

Using the combinatorial interpretations of $\vartheta\left(\left(\lambda_{i-1}\right)_{\varphi_{i}^{-1}(j)}\right)$ and $\vartheta\left(\lambda_{l}, \tau ; \rho\right)$, and the notion of the composite permutation, we may therefore write $c_{\lambda,(d),\{\tau\},\{\rho\}}$ as a sum over the set $S_{\lambda, \tau, \rho}$. To remove the dependence on the chosen orders, we will average over all choices of them.

We note that any composite permutation has the property that the last symbol of any kind $j \in I(\lambda)$ is not immediately followed by the first symbol of some kind $j^{\prime} \in I(\tau)$. Also, with the natural induced ordering, any composite partition satisfies condition (1) in the Main Claim.

\section{E.c. Simplification}

For any permutation in $S_{\lambda, \tau, \rho}$, we assign a set partition $P \vdash I(\lambda)$, which measures in what way it fails to satisfy condition (2) in the Main Claim. We define $P$ to be the finest set partition such that if the last symbol of kind $i$ is immediately followed by a symbol of kind $j$ for $i, j \in I(\lambda)$ then $i$ and $j$ lie in the same set of $P$.

Now, suppose we are given a chain of partitions $\lambda, \lambda_{1}, \ldots, \lambda_{l}$ along with additional refining data, orderings and base permutation $S_{l}$ as above. Let $P$ be the failure set partition of the composite permutation. Let $\lambda_{l+1}:=\lambda^{P}$ be the partition formed by merging parts of $\lambda$ according to $P$, so there is a canonical refining function $I(\lambda) \rightarrow I\left(\lambda^{P}\right)$. By the construction of the composite permutation, this function actually factors through a refining function $\varphi^{\prime}: I\left(\lambda_{l}\right) \rightarrow I\left(\lambda_{l+1}\right)$. Suppose that this refining function $\varphi^{\prime}$ is nontrivial, i.e. $\lambda_{l} \neq \lambda^{P}$.

We note that if we change the order on $I\left(\lambda_{l}\right) \sqcup I(\tau)$ such that the order on $I(\tau)$ and each inverse image of $\varphi^{\prime}$ is preserved, the failure partition $P$ does not change. We will therefore group these orderings together.

On the other hand, consider the following data:

- the chain $\lambda, \lambda_{1}, \ldots, \lambda_{l}, \lambda_{l+1}$ with refining maps $\varphi_{i}$ and $\varphi^{\prime}$ as before,

- the orders and refining data corresponding to the $\varphi_{i}$ as before, 
- in addition, an order on each preimage of $\varphi^{\prime}$ which is induced by the order on $I\left(\lambda_{l}\right) \sqcup I(\tau)$,

- refining data corresponding to $\varphi^{\prime}$ induced from the permutation corresponding to $\lambda_{l}, \tau$ and $\rho$,

- any order on $I\left(\lambda_{l+1}\right) \sqcup I(\tau)$ such that the restriction to $I(\tau)$ is the restriction of the order on $I\left(\lambda_{l}\right) \sqcup I(\tau)$ and such that elements of $I(\tau)$ appear before elements of $I\left(\lambda_{l+1}\right)$,

- permutations of $\left(\lambda_{l+1}\right)_{i}+1, \tau_{i}+1, \rho_{i}$ symbols of kind $i$ for $i \in I\left(\lambda_{l+1}\right), i \in I(\tau)$ and $i \in I(\rho)$ respectively, defined from the permutation corresponding to $\lambda_{l}$ by leaving out the last symbol of any kind $i \in I\left(\lambda_{l}\right)$ which is not the last one in a level set of $\varphi^{\prime}$ and identifying symbols according to $\varphi^{\prime}$.

It is easy to check that the refining data and the permutation still satisfy the order conditions. Furthermore, the failure set partition of the composite partition of this new data is still $P$, so that now $\lambda_{l+1}=\lambda^{P}$.

The original chain with additional data giving failure set partition $P$ and the extended chain with additional data contribute to $c_{\lambda,(d),\{\tau\},\{\rho\}}$ in formula (3) with opposite signs since the extended chain is one element longer. We claim these contributions cancel.

For the original chain, we have

$$
\frac{\left(\ell\left(\lambda_{l}\right)\right) !}{\prod_{j \in I\left(\lambda_{l+1}\right)}\left|\varphi^{\prime-1}(j)\right| !}
$$

choices of orders on $I\left(\lambda_{l}\right) \sqcup I(\tau)$ in the above construction. For the extended chain, we made

$$
\left|\operatorname{Aut}\left(\lambda_{l+1}\right)\right|\left(\ell\left(\lambda_{l+1}\right)\right) !
$$

choices in the above construction.

However, the contributions are also weighted by averaging over choices of orders and by the automorphism factors in (3). For the original chain, the weight is

$$
\left(\left(\ell\left(\lambda_{l}\right)\right) !\right)^{-1}
$$

and for the extended chain, the weight is

$$
\left(\left|\operatorname{Aut}\left(\lambda_{l+1}\right)\right|\left(\ell\left(\lambda_{l+1}\right)\right) ! \prod_{j \in I\left(\lambda_{l+1}\right)}\left|\varphi^{\prime-1}(j)\right| !\right)^{-1} .
$$

Thus, the two contributions cancel.

The only remaining contributions occur when $l=0$ and $P$ is the set partition into one-element sets. Since they are weighted with coefficient one, this finishes the proof of the Main Claim.

\section{F. Proof of the Housing Theorem}

We begin with a simple lemma.

Lemma 4. Suppose $r+s+\ell(\gamma)<\ell(\lambda)$. Then $c_{\lambda, \gamma,\left\{\tau_{i}\right\},\left\{\rho_{i}\right\}}=0$.

Proof. We examine the summand in formula (2) corresponding to some $\varphi$. A factor in this summand can only be nonzero if $r(i)+s(i)+1 \geq \ell\left(\varphi^{-1}(i)\right)$. Therefore each summand will vanish unless $r+s+\ell(\gamma) \geq \ell(\lambda)$.

Now let us suppose that $\left(\gamma,\left\{\tau_{i}\right\},\left\{\rho_{i}\right\}\right)$ is the housing data of a boundary class of the generating set. We need to show that $c_{\lambda, \gamma,\left\{\tau_{i}\right\},\left\{\rho_{i}\right\}}=0$ for each $\lambda$ which is not a housing partition.

Let us first study the case $\ell(\lambda)>2 g-2-d$. Since $\gamma$ is derived from a boundary stratum of codimension at most $2 g-3-d-r-s$ (we are missing the $\psi$ - and $\mathcal{k}$-classes from the components, which do not contribute to $\gamma$ ) by diminishing parts by their $\kappa$ - and $\psi$-degrees, we have the inequality $\ell(\gamma) \leq 2 g-2-d-r-s$. Then 
by Lemma 4 we are done in this case. The same argument settles also the case where there are components of the boundary stratum we are considering which do not appear in $\gamma$ and $\ell(\lambda)=2 g-2-d$.

Now assume that $\ell(\lambda)=2 g-2-d$ and that $\lambda$ contains no even part. Then by the same arguments if the coefficient is nonzero, we must have $\ell(\gamma)=2 g-2-d-r-s$. Furthermore, from the proof of Lemma 4 we see that $r(i)+s(i)=\ell\left(\varphi^{-1}(i)\right)-1$ for each $i \in I(\gamma)$. This implies $\ell\left(\varphi^{-1}(i)\right)+r(i)+s(i) \equiv 1(\bmod 2)$, and therefore for each $i \in I(\gamma)$, we have $\gamma_{i}+r(i)+s(i) \equiv 1(\bmod 2)$. Hence each part of the housing data (for the underlying boundary stratum), which $\gamma$ was obtained from by subtraction of $r(i)+s(i)$ from each part, is odd. This is a contradiction, so the coefficient must be zero, as desired.

\section{The Rank Theorem}

\section{A. Reformulation}

Let us first formulate a stronger version of the Rank Theorem.

Theorem 2. For any $\mathcal{\kappa}$-polynomial $F$ in degree $r:=2 g-3-d$, the following two statements are equivalent:

(1) For any $\pi \in P(g-2-r)$, we have $\int_{\bar{M}_{g}} F \kappa_{\pi} \lambda_{g} \lambda_{g-1}=0$.

(2) There is a $B \in P B R^{r}\left(M_{g}^{c}\right)$ such that for any $\pi^{\prime} \in P(2 g-3-r)$ we have $\int_{\bar{M}_{g}}(F-B) \kappa_{\pi^{\prime}} \lambda_{g}=0$.

It will be convenient to show that we can replace the first condition in Theorem 2 by

(3) For any $\pi \in P(g-2-r)$ of length at most $r+1$ we have $\int_{\bar{M}_{g}} F \kappa_{\pi} \lambda_{g} \lambda_{g-1}=0$.

Then Theorem 2 will follow from the following simple argument. Consider an $F$ satisfying the second condition and we want to show that $\int_{\bar{M}_{g}} F \kappa_{\pi} \lambda_{g} \lambda_{g-1}=0$ for some given $\pi \in P(g-2-r)$. Notice that then also $F \kappa_{\pi}$ satisfies the second condition since $B \kappa_{\pi}$ lies in $B R^{g-2}\left(M_{g}^{c}\right)$ and by the Housing Theorem can be replaced by some $B^{\prime} \in P B R^{g-2}\left(M_{g}^{c}\right)$. We then find that $\int_{\bar{M}_{g}} F \kappa_{\pi} \lambda_{g} \lambda_{g-1}=0$ since in this case the length condition is trivial.

Let $v_{F} \in\left(\mathbb{Q}^{P(d)}\right)^{*}$ be defined by the $\lambda_{g}$-pairing of $F$ with the kappa ring. The linear forms $M_{\lambda}$ (defined in Section 3.B) form a basis for $\left(\mathbb{Q}^{P(d)}\right)^{*}$, so $v_{F}$ can be written as a linear combination of them. The second condition in the theorem is then equivalent to the condition that only the $M_{\lambda}$ coming from actual boundary strata (those where $\lambda$ is a housing partition) are used in this expansion of $F$. Notice that by Lemma 4 we can assume that the $\lambda$ are partitions of $2 g-3-r$ of length at most $r+1$. By Lemma 2, this means that the only non-housing partitions that might occur are of length exactly $r+1$ with only odd parts. For the proof of the Rank Theorem we will need to understand the coefficients corresponding to these partitions better.

Observe that partitions of $2 g-3-r$ of length exactly $r+1$ with only odd parts correspond to partitions of $g-2-r$ of length at most $r+1$. So for any $\sigma \in P(g-2-r, r+1)$ we can look at $\eta_{\sigma}, \mu_{\sigma} \in\left(\mathbb{Q}^{P(r)}\right)^{*}$ with $\eta_{\sigma}(\tau):=c_{\lambda,(d),\{\tau\},\{\emptyset\}}$, where $\lambda$ is the partition of $2 g-3-r$ of length $r+1$ corresponding to $\sigma$, and $\mu_{\sigma}(\tau)$ is up to a factor the integral $\int_{\bar{M}_{g}} \kappa_{\sigma} \kappa_{\tau} \lambda_{g} \lambda_{g-1}$, namely

$$
\mu_{\sigma}(\tau)=\sum_{P \vdash I(\sigma) \sqcup I(\tau)}(-1)^{\ell(\sigma)+\ell(\tau)+|P|} \frac{(2 g-3+|P|) !}{\prod_{i \in P}\left(2(\sigma, \tau)_{i}^{P}+1\right) ! !} .
$$

So what we need to show is the following:

Claim. The $\mathbb{Q}$-subspaces of $\left(\mathbb{Q}^{P(r)}\right)^{*}$ spanned by $\eta_{\sigma}$ and $\mu_{\sigma}$ for $\sigma$ ranging over all partitions of $g-2-r$ of length at most $r+1$ are equal.

Recall from Section 3.E.e that $\eta_{\sigma}(\tau)$ is the number of all permutations $S$ of $\lambda_{i}+1$ symbols of kind $i \in I(\lambda)$ and $\tau_{i}+1$ symbols of kind $i \in I(\tau)$ satisfying 
(1) The last symbol of kind $i$ for some $i \in I(\lambda)$ is either at the end of the sequence or immediately followed by a symbol of kind $j$ for some $j \in I(\tau)$ which is not the first of its kind.

(2) The successor of the last element of kind $i$ is not the first element of kind $j$ for any $i, j \in I(\tau)$ with $i<j$, where we fix some order on $I(\tau)$.

Before coming to the main part of the proof we apply an invertible transformation $\Phi$ to $\left(\mathbb{Q}^{P(r)}\right)^{*}$ to simplify the definitions of $\eta$ and $\mu$. The inverse of the transformation we want to apply sends a linear form $\varphi^{\prime} \in\left(\mathbb{Q}^{P(r)}\right)^{*}$ to the linear form $\varphi$ defined by

$$
\varphi(\tau)=\sum_{P \vdash I(\tau)}(-1)^{\ell(\tau)+|P|} \varphi^{\prime}\left(\tau^{P}\right) .
$$

The transformation $\Phi$ defined in this way is clearly invertible. By a similar argument as in the proof of Lemma 3, we can show that the image $\eta_{\sigma}^{\prime}$ of $\eta_{\sigma}$ under $\Phi$ is defined in the same way as $\eta_{\sigma}$ but leaving out Condition (2) on the permutations.

To study the action of $\Phi$ on $\mu$, we use the following lemma:

Lemma 5. Let $F$ be a function $F: P(n+m) \rightarrow \mathbb{Q}$ and define for any $\sigma \in P(n)$ functions $G_{\sigma}, G_{\sigma}^{\prime}: P(m) \rightarrow \mathbb{Q}$ in terms of $F$ by

$$
\begin{aligned}
& G_{\sigma}(\tau)=\sum_{P \vdash I(\sigma) \sqcup I(\tau)} F\left((\sigma \sqcup \tau)^{P}\right) \\
& G_{\sigma}^{\prime}(\tau)=\sum_{\substack{P \vdash I(\sigma) \sqcup I(\tau) \\
P \text { separates } I(\tau)}} F\left((\sigma \sqcup \tau)^{P}\right),
\end{aligned}
$$

where the second sum just runs over set partitions $P$ such that each element of $I(\tau)$ belongs to a separate part. Then

$$
G_{\sigma}(\tau)=\sum_{P \vdash I(\tau)} G_{\sigma}^{\prime}\left(\tau^{P}\right) .
$$

Proof. Given set partitions $P$ of $I(\tau)$ and $Q$ of $I(\sigma) \sqcup I\left(\tau^{P}\right)$, with $Q$ separating $I\left(\tau^{P}\right)$, we can alter $Q$ by replacing each element of $I\left(\tau^{P}\right)$ by the elements in the corresponding part of $P$. Each set partition of $I(\sigma) \sqcup I(\tau)$ is obtained exactly once by this construction.

Using this lemma and keeping track of the sign factors, we have that $\mu_{\sigma}^{\prime}(\tau)$ is

$$
\mu_{\sigma}^{\prime}(\tau)=\sum_{\substack{P \vdash I(\sigma) \sqcup I(\tau) \\ P \text { separates } I(\tau)}}(-1)^{\ell(\sigma)+\ell(\tau)+|P|} \frac{(2 g-3+|P|) !}{\prod_{i \in P}\left(2(\sigma \sqcup \tau)_{i}^{P}+1\right) ! !} .
$$

We can use the lemma again with the roles of $\sigma$ and $\tau$ interchanged to replace the generators of the span of $\mu_{\sigma}^{\prime}$ by $\mu_{\sigma}^{\prime \prime}$ with

$$
\mu_{\sigma}^{\prime \prime}(\tau):=\sum_{\substack{P \vdash I(\sigma) \sqcup I(\tau) \\ P \text { separates } I(\tau) \\ P \text { separates } I(\sigma)}}(-1)^{\ell(\sigma)+\ell(\tau)+|P|} \frac{(2 g-3+|P|) !}{\prod_{i \in P}\left(2(\sigma \sqcup \tau)_{i}^{P}+1\right) ! !} .
$$

Therefore we have reduced the proof of the Rank Theorem to proving the following claim.

Claim. The $\mathbb{Q}$-subspaces of $\left(\mathbb{Q}^{P(r)}\right)^{*}$ spanned by $\eta_{\sigma}^{\prime}$ and $\mu_{\sigma}^{\prime \prime}$ for $\sigma$ ranging over all partitions of $g-2-r$ of length at most $r+1$ are equal. 


\section{B. Further strategy of proof}

In order to prove the claim we will establish interpretations for $\eta_{\sigma}^{\prime}(\tau)$ and $\mu_{\sigma}^{\prime \prime}(\tau)$ as counts of symbols of different kinds satisfying some ordering constraints. This enables us to find nonzero constants $F(i)$ for each $i \in I(\sigma)$ independent of $\tau$ such that

$$
\mu_{\sigma}^{\prime \prime}(\tau)=\sum_{P \vdash I(\sigma)} \prod_{i \in P} F(i) \frac{\eta_{\sigma^{P}}^{\prime}(\tau)}{(r+1-|P|) !},
$$

giving a triangular transformation.

For the interpretations the notion of a comb-like order plays an important role. We say that symbols $i_{1} \ldots i_{2 m+1}$ are in comb-like order if we have the relations $i_{1}<i_{3}<\cdots<i_{2 m+1}$ and $i_{2 j}<i_{2 j+1}$ for $j \in[m]$. This is illustrated in Figure 1.

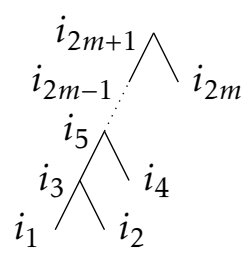

Figure 1: A comb-like order

Note that the number of comb-like orderings of $2 m+1$ symbols is $(2 m+1) ! /(2 m+1) ! !$. More generally the number

$$
\frac{(2|\pi|+\ell(\pi)) !}{\prod_{i \in I(\pi)}\left(2 \pi_{i}+1\right) ! !}
$$

corresponding to a partition $\pi$ counts the number of permutations of the $2|\pi|+\ell(\pi)$ symbols $\bigcup_{i \in I(\pi)}\left\{i_{1}, \ldots\right.$, $\left.i_{2 \pi_{i}+1}\right\}$ such that symbols corresponding to the same part of $\pi$ appear in comb-like order.

\section{C. Combing orders}

We obtain a first reinterpretation of $\eta_{\sigma}^{\prime}(\tau)$ by numbering the symbols of the same kind:

Interpretation A1. $\eta_{\sigma}^{\prime}(\tau)$ is the number of all permutations of symbols $i_{1}, \ldots, i_{\tau_{i}+1}$ for $i \in I(\tau)$ and $i_{1}, \ldots, i_{\lambda_{i}+1}$ for $i \in I(\lambda)$ such that for fixed $i \in I(\tau) \sqcup I(\lambda)$ the $i_{j}$ appear in order and for all $i \in I(\lambda)$ the symbol $i_{\lambda_{i}+1}$ is either at the end of the sequence or immediately followed by some $j_{k}$ for $j \in I(\tau)$ and $k \neq 1$.

Since $\lambda$ has length $r+1$ and $|\tau|=r$, such a permutation gives a bijection between the $j_{k}$ for $j \in I(\tau)$ with $k \neq 1$ and all but one of the $i_{\lambda_{i}+1}$ for $i \in I(\lambda)$. After picking this bijection, we can remove the $i_{\lambda_{i}+1}$.

Interpretation $A 2 . \quad \eta_{\sigma}^{\prime}(\tau)$ is the sum over bijections

$$
\varphi: I(\lambda) \rightarrow\left\{i_{j} \mid i \in I(\tau), j \neq 1\right\} \sqcup\{\text { End }\}
$$

of the number of permutations of symbols $i_{1}, \ldots, i_{\tau_{i}+1}$ for $i \in I(\tau)$ and $i_{1}, \ldots, i_{\lambda_{i}}$ for $i \in I(\lambda)$ such that symbols of the same kind appear in order and all symbols $i_{j}$ for $i \in I(\lambda)$ appear before $\varphi(i)$ (this condition is empty if $\varphi(i)=$ End).

We can then add new symbols immediately following each $i_{\lambda_{i}}$ for $i \in I(\lambda)$ and reindex the $i_{j}$ for $i \in I(\tau)$ to create comb-like orderings. 
Interpretation A3. $\eta_{\sigma}^{\prime}(\tau)$ is the sum over bijections

$$
\varphi: I(\lambda) \rightarrow\left\{i_{j} \mid i \in I(\tau), j \text { even }\right\} \sqcup\{\text { End }\}
$$

of the number of permutations of symbols $i_{1}, \ldots, i_{2 \tau_{i}+1}$ for $i \in I(\tau), i_{1}, \ldots, i_{\lambda_{i}}$ for $i \in I(\lambda)$, and an additional symbol End such that the $i_{j}$ for $i \in I(\tau)$ appear in comb-like order, the $i_{j}$ for $i \in I(\lambda)$ appear in order, and $i_{\lambda_{i}}$ for $i \in I(\lambda)$ is immediately followed by $\varphi(i)$.

Recall that $\lambda$ is defined in terms of $\sigma$ by taking the numbers $2 \sigma_{i}+1$ for each $i \in I(\sigma)$ and adding as many ones as needed to reach length $r+1$. There is only one symbol $i_{1}$ of kind $i$ for $i \in I(\lambda) \backslash I(\sigma)$ in Interpretation $\mathrm{A} 3$ of $\eta_{\sigma}^{\prime}(\tau)$ and it must be immediately followed by $\varphi(i)$. For convenience set $(r+1-\ell(\sigma))$ ! $\eta_{\sigma}^{\prime \prime}:=\eta_{\sigma}^{\prime}$. Removing these symbols $i_{1}$ gives an interpretation of $\eta_{\sigma}^{\prime \prime}$.

Interpretation A4. $\eta_{\sigma}^{\prime \prime}(\tau)$ is a sum over all injections

$$
\varphi: I(\sigma) \rightarrow\left\{i_{j} \mid i \in I(\tau), j \text { even }\right\} \sqcup\{\text { End }\}
$$

of the number of permutations of symbols $i_{1}, \ldots, i_{2 \tau_{i}+1}$ for $i \in I(\tau), i_{1}, \ldots, i_{2 \sigma_{i}+1}$ for $i \in I(\sigma)$, and an additional symbol End such that the $i_{j}$ for $i \in I(\tau)$ appear in comb-like order, the $i_{j}$ for $i \in I(\sigma)$ appear in order, and $i_{2 \sigma_{i}+1}$ for $i \in I(\sigma)$ is immediately followed by $\varphi(i)$.

We now switch to the interpretation of $\mu_{\sigma}^{\prime \prime}(\tau)$, which was defined in (4). The coefficient corresponding to a set partition $P$ can be interpreted as the number of permutations of symbols $i_{1}, \ldots, i_{2(\sigma \sqcup \tau)_{i}^{P}+1}$ for $i \in I\left((\sigma \sqcup \tau)^{P}\right)$ and one additional symbol $\star$ such that all $i_{1}, \ldots, i_{2(\sigma \sqcup \tau)_{i}^{P}+1}$ for $i \in I\left((\sigma \sqcup \tau)^{P}\right)$ appear in comb-like order.

Because of the restrictions in the sum, the parts of $P$ are either singletons or contain exactly one element from each of $I(\sigma)$ and $I(\tau)$. This defines a function $\psi: I(\sigma) \rightarrow I(\tau) \sqcup\{\star\}$, injective when restricted to the preimage of $I(\tau)$. Interpreting the summands as counting comb-like orders and cutting combs into two pieces for each part of $P$ of size two gives the following:

Interpretation B1. $\mu_{\sigma}^{\prime \prime}(\tau)$ is the sum over functions

$$
\psi: I(\sigma) \rightarrow I(\tau) \sqcup\{\star\}
$$

such that $\left.\psi\right|_{\psi^{-1}(I(\tau))}$ is injective, of a sign of $(-1)^{\left|\psi^{-1}(I(\tau))\right|}$ times the number of permutations of symbols $i_{1}, \ldots, i_{2 \tau_{i}+1}$ for $i \in I(\tau), i_{1}, \ldots, i_{2 \sigma_{i}+1}$ for $i \in I(\sigma)$ and one additional symbol $\star$ such that all $i_{1}, \ldots, i_{2 \tau_{i}+1}$ for $i \in I(\tau)$ and all $i_{1}, \ldots, i_{2 \sigma_{i}+1}$ for $i \in I(\sigma)$ appear in comb-like order and such that $i_{2 \sigma_{i}+1}$ for $i \in I(\sigma)$ with $\psi(i) \neq \star$ is immediately followed by $\psi(i)_{1}$.

Now we split the set of such permutations depending on the symbols immediately following symbols $i_{2 \sigma_{i}+1}$ for $i \in I(\sigma)$. We notice that the signed sum exactly kills those permutations where some $i_{2 \sigma_{i}+1}$ for $i \in I(\sigma)$ is immediately followed by some $j_{1}$ for $j \in I(\tau)$ since if such a summand appears for some $\psi$ with $\psi(i) \neq j$ we must have $\psi(i)=\star$ and we find the same summand with opposite sign in the sum corresponding to the map $\psi^{\prime}$ defined by $\psi^{\prime}(i)=j$ and $\psi^{\prime}(k)=\psi(k)$ for $k \neq i$ and vice versa.

Interpretation B2. $\mu_{\sigma}^{\prime \prime}(\tau)$ is the number of permutations of symbols $i_{1}, \ldots, i_{2 \tau_{i}+1}$ for $i \in I(\tau), i_{1}, \ldots, i_{2 \sigma_{i}+1}$ for $i \in I(\sigma)$ and one additional symbol $\star$ such that all $i_{1}, \ldots, i_{2 \tau_{i}+1}$ for $i \in I(\tau)$ and all $i_{1}, \ldots, i_{2 \sigma_{i}+1}$ for $i \in I(\sigma)$ appear in comb-like order and such that $i_{2 \sigma_{i}+1}$ for $i \in I(\sigma)$ is not immediately followed by a symbol of the form $j_{1}$ with $j \in I(\tau)$.

Interpretations A4 and B2 are very close. The differences between the two of them are that the $\sigma$-type symbols are in total order rather than comb-like order in A4 and that the conditions on the elements immediately following the $i_{2 \sigma_{i}+1}$ are different. 
We now break $\mu_{\sigma}^{\prime \prime}(\tau)$ into a sum over set partitions $P$ of $I(\sigma)$. Given a permutation of the symbols appearing in Interpretation B2, define a function

$$
\varphi: I(\sigma) \rightarrow\left\{i_{j} \mid i \in I(\tau), j \text { even }\right\} \sqcup\{\text { End }\}
$$

recursively by

$$
\varphi(i)=\left\{\begin{array}{rr}
j_{2 k} \quad \begin{array}{r}
\text { if } i_{2 \sigma_{i}+1} \text { for } i \in I(\sigma) \text { is immediately followed by a symbol } \\
\text { of the form } j_{2 k} \text { or } j_{2 k+1} \text { with } j \in I(\tau),
\end{array} \\
\varphi(j) \quad \begin{array}{r}
\text { if } i_{2 \sigma_{i}+1} \text { for } i \in I(\sigma) \text { is immediately followed by a symbol } \\
\text { of the form } j_{k} \text { with } j \in I(\sigma) .
\end{array}
\end{array}\right.
$$

Then let $P$ be the set partition of preimages under $\varphi$. We will identify the summand of $\mu_{\sigma}^{\prime \prime}(\tau)$ corresponding to such a set partition $P$ as $\eta_{\sigma^{P}}^{\prime \prime}(\tau)$ times a factor depending only on $\sigma$ and $P$.

This factor is equal to

$$
\prod_{i \in P} F(i)
$$

where

$$
F(i)=\frac{\left(2 \sigma_{i}^{P}+|i|+1\right) !}{\prod_{j \in i}\left(2 \sigma_{j}+1\right) ! !} .
$$

Here, $F(i)$ should be interpreted as the number of permutations of $2 \sigma_{j}+1$ symbols of kind $j$ for each $j \in i$ and one additional symbol End such that the symbols of each kind appear in comb-like order. If these permutations are interpreted as refinement data, then the permutations counted by the $P$-summand of $\mu_{\sigma}^{\prime \prime}(\tau)$ are the refinements by this data of the permutations counted by $\eta_{\sigma^{P}}^{\prime \prime}(\tau)$.

Thus we have the identity

$$
\mu_{\sigma}^{\prime \prime}=\sum_{P \vdash I(\sigma)} \prod_{i \in P} F(i) \eta_{\sigma^{P}}^{\prime \prime}
$$

This is a triangular change of basis with nonzero entries on the diagonal, so the $\mu^{\prime \prime}$ and $\eta^{\prime \prime}$ span the same subspace in $\left(\mathbb{Q}^{P(r)}\right)^{*}$. This completes the proof of the Rank Theorem.

\section{References}

[1] C. Faber, A conjectural description of the tautological ring of the moduli space of curves. In: Moduli of curves and abelian varieties, pp. 109-129, Aspects Math., E33, Vieweg, Braunschweig, 1999. MR-1722541

[2] C. Faber and R. Pandharipande, Hodge integrals and Gromov-Witten theory, Invent. Math. 139 (2000), no. 1, 173-199. MR-1728879

[3] C. Faber and R. Pandharipande, Hodge integrals, partition matrices, and the $\lambda_{g}$ conjecture, Ann. of Math. (2) 157 (2003), no. 1, 97-124. MR-1954265

[4] E. Getzler and R. Pandharipande, Virasoro constraints and the Chern classes of the Hodge bundle, Nuclear Phys. B 530 (1998), no. 3, 701-714. MR-1653492

[5] T. Graber and R. Pandharipande, Constructions of nontautological classes on moduli spaces of curves, Michigan Math. J. 51 (2003), no. 1, 93-109. MR-1960923

[6] R. Pandharipande, The $\varkappa$ ring of the moduli of curves of compact type, Acta Math. 208 (2012), no. 2, 335-388. MR-2931383 
[7] R. Pandharipande and A. Pixton, Relations in the tautological ring of the moduli space of curves, preprint 2013. arXiv:1301.4561

[8] D. Petersen, Tautological rings of spaces of pointed genus two curves of compact type, Compos. Math. 152 (2016), no. 7, 1398-1420. MR-3530445 